\title{
RIEMANN DERIVATIVES AND GENERAL INTEGRALS
}

\author{
S. De Sarkar and A.G. Das
}

Sargent and later Bullen and Mukhopadhyay obtained a definition of absolutely continuous functions, $A C_{k}^{*}$ functions, that is related to $k$ th Peano derivatives. The generalised notions of $A C_{k} G^{*},\left[A C_{k} G^{*}\right], A C_{k} G^{*}$ above, etcetera functions led Bullen and Mukhopadhyay to define certain general integrals of the $k$ th order.

The present work is concerned with a further simplification of the definitions of such functions by the use of divided differences but still retaining similar fundamental properties. These concepts lead to the introduction of Denjoy and Ridder type integrals which are shown to be equivalent to a Perron type integral that corresponds to $k$ th Riemann* derivatives. All three of these integrals are shown to be equivalent to the three integrals of Bullen and Mukhopadhyay.

\section{Introduction.}

A fuction of a real variable $f$ is $(L)$ integrable if there exists a function $F$ such that

(i) $F^{\prime}(x)=f(x)$ at almost all points $x$ and

Received 18 March 1986.

Copyright Clearance Centre, Inc. Serial-fee code: 0004-9729/87 $\$ A 2.00+0.00$. 
(ii) $F$ is absolutely continuous.

The function $F$ is called the indefinite $(L)$ integral of $f$. If the condition ( $i$ ) is replaced by the condition that $F$ is $A C G_{*}$, we obtain the definition of the $\left(D^{*}\right)$ integral, the special Denjoy integral.

It is well known that if a function $F$ has a finite derivative on a set $E$ on which $F$ is continuous, then $F$ is $A C G_{*}$ on $E$ and that if $F$ is $A C G_{*}$ on a set $E$ then a finite derivative of $F$ exists almost everywhere in $E$. This fact enables us to show that the $\left(D^{*}\right)$ integral is equivalent to the $(P)$ integral of Perron. Later Ridder [14] obtained the generalisations $A C G_{*}$ above and $A C G_{\text {* }}$ below functions leading to the definition of the $(P)$ integral, say, which is again completely equivalent to the $\left(D^{*}\right)$ and $(P)$ integrals.

Das and Lahiri [8] obtained the definition of a $k$ th absolutely continuous function, $A C_{k}$ function. Das and Das [7] showed that the first integral of an $A C_{k}$ function is $A C_{k+1}$, and that a $k$-fold (L) integral is an $A C_{k}$ function.

An equivalent descriptive definition of a $k$-fold integral given by them is as follows:

A function of a real variable $f$ is $\left(L^{k}\right)$ integrable on $[a, b]$ if there exists a function $F$ such that

(i) $F^{(k)}(x)=f(x)$ at almost all points $x$ in $[a, b]$ and (ii) $F$ is $A C_{k}$ on $[a, b]$.

The function $F$ (thus uniquely determined apart from a polynomial of degree $k-1)$ is the indefinite $\left(L^{k}\right)$ integral of the function $f$.

In order to obtain the $k$ th order generalisation of the special Denjoy and Ridder integrals it is desirable to introduce generalisations of the concepts of $A C G_{*}, A C G_{*}$ above and $A C G_{*}$ below functions.

The first part of this paper is concerned with such extensions giving rise to the definitions of $A C_{k}^{*}, A C_{k}^{*} G, A C_{k}^{*} G$ above and $A C_{k}^{*} G$ below functions. It is proved that if the $k$ th Riemann* derivative of $f, D^{k} f$, exists in $[a, b]$ then $f$ is $A C_{k}^{*} G$ on $[a, b]$ and that if $f$ 
is $A C_{k}^{*} G$ on $[a, b]$ then $D^{k} f$ exists and is equal to the approximate derivative of $D^{k-1}$ almost everywhere in $[a, b]$.

The second part introduces the definitions of the $P^{k}-, D^{k}-$ and $p^{k}$ - integrals which are ultimately shown to be equivalent.

It is worth mentioning that our definitions of $A C_{k}^{*}, A C_{k}^{*} G$ etcetera functions are simpler than those in sargent [20] and Bullen and Mukhopadhyay [3] but still retain similar fundamental properties. Also our integrals are shown to be equivalent to the $p^{k}-, D^{k}$ - and $p^{k}-$ integrals in Bullen [2] and Bullen and Mukhopadhyay [3]. It is thus evident that a function $f$ is $P^{k}$ - integrable if and only if it is $C_{k-1} P$ - integrable and

$$
P^{k}-\int_{a}^{x} f=(P) \int_{a}^{x} c_{1} P-\int_{a}^{x} \ldots c_{k-1} P-\int_{a}^{x} f,
$$

a result that the extension of the $\left(L^{k}\right)$ integral actually has.

Let $f$ be a real valued function defined on the real line $R_{1}$. Let $a, b$ be fixed real numbers such that $a<b$. Let $k$ be a positive integer greater than 1 . By $E$ we shall always mean a subset of $[a, b]$. We remark here that the set $E$ which was taken to be dense in itself in De Sarkar and Das [10] may be taken as any subset of $[a, b]$, but in that case the results connected with the existence of derivatives should be modified for the points of the derived set $E^{\prime}$ which are in $E$. We denote by $\alpha, \beta$ the least upper bound and the greatest lower bound of $E$ respectively. The Lebesgue measure of a set $A$ will be denoted by $m A$. The usual $k$ th order derivative and the approximate derivative of $f$ at $x, x \in R_{1}$, will be denoted by $f^{(k)}(x)$ and $f_{o p}^{\prime}(x)$ respectively; $f_{k}(x)$ will stand for the $k$ th order Peano derivative of $f$ at $x$.

Let $x_{o}, x_{1}, \ldots, x_{r}$ be any $r+1$ distinct points, not necessarily in linear order, in $[a, b]$. Then the rth divided difference of $f$ is defined by 


$$
Q_{r}\left(f ; x_{0}, x_{1}, \ldots, x_{r}\right)=\sum_{i=0}^{r}\left\{f\left(x_{i}\right) / \prod_{\substack{j=0 \\ j \neq i}}^{r}\left(x_{i}-x_{j}\right)\right\} .
$$

Let $x, x_{0}, \ldots, x_{r}$ be points of $[a, b]$ and let $h_{i}=x_{i}-x$ $i=0,1, \ldots, r$ with

$$
0 \leq\left|h_{0}\right|<\left|h_{1}\right|<\ldots<\left|h_{r}\right|
$$

Then the rth Riemann derivative of $f$ at $x$ is defined by $D^{r} f(x)=r ! \lim _{h_{r} \rightarrow 0} \lim _{h_{p-1} \rightarrow 0} \cdots \lim _{h_{0} \rightarrow 0} Q_{r}\left(f ; x_{0}, x_{1}, \ldots, x_{r}\right)$,

if the iterated limit exists. The right and the left rth Riemann derivatives are defined in the obvious way. If in the above definition $h_{0}=0$, then we get the rth Riemann* derivative of $f$ at $x$ and denote it by $D^{r} f(x)$. Taking lim sup (respectively lim inf) at each stage we get the upper (respectively lower) derivatives $\bar{D}^{r} f(x), \vec{D}^{2} f(x)$ (respectively $\left.\underline{D}^{r} f(x), \underline{D}^{r} f(x)\right)$. The one sided derivatives $D_{+}^{r} f(x)$, $D_{+}^{r} f(x)$ etcetera are obtained in the usual way.

Let $E \subset[a, b]$. If, for all choices of $k+1$ distinct points $x_{0}, x_{1}, \ldots, x_{k}$ in $E$, we have $Q_{k}\left(f ; x_{0}, x_{1}, \ldots, x_{k}\right) \geq 0$, then $f$ is called $k$ convex on $E$.

The number

$$
v_{k}(f ; E)=\sup _{\pi} \sum_{i=0}^{n-k}\left(x_{i+k}-x_{i}\right)\left|Q_{k}\left(f ; x_{i}, \ldots, x_{i+k}\right)\right|
$$

where the supremum is taken over all $\pi$-subdivisions in $E$ of the form $x_{0}<x_{1}<\ldots<x_{n}, x_{i} \in E, i=0,1, \ldots, n$, is called the $k t h$ variation of $f$ on $E$. If $V_{k}(f ; E)<+\infty$, then $f$ is said to be of bounded kth variation, $B V_{k}$, on $E$.

$$
\text { Let } x_{1,0}<x_{1,1}<\ldots<x_{1, k} \leq x_{2,0}<x_{2,1}<\ldots<x_{2, k} \leq \ldots
$$

$\leq x_{n, 0}<x_{n, 1}<\ldots<x_{n, k}$ be any subdivision of $E$ where $x_{i, j} \in E$. We say that the intervals $\left(x_{i, 0}, x_{i, k}\right), i=1,2, \ldots, n$, form an 
elementary system $I$, say, in $E$. The system is denoted by

$$
I\left(x_{i, 1}, \ldots, x_{i, k-1}\right):\left(x_{i, 0}, x_{i, k}\right), i=1,2, \ldots, n \text {. }
$$

We write

$$
\begin{aligned}
\sigma|I| & =\sum_{i=1}^{n}\left(x_{i, k}-x_{i, 0^{\prime}}\left|Q_{k}\left(f ; x_{i, 0}, \ldots, x_{i, k}\right)\right|,\right. \\
\sigma I & =\sum_{i=1}^{n}\left(x_{i, k}-x_{i, 0^{\prime}}\right) Q_{k}\left(f ; x_{i, 0}, \ldots, x_{i, k}\right)
\end{aligned}
$$

and

$$
m I=\sum_{i=1}^{n}\left(x_{i, k^{-x}} i, 0^{\prime}\right.
$$

The function $f$ is said to be absolutely kth continuous, $A C_{k}$, on $E$ if $(\forall E>0)(Z \delta(\varepsilon)>0)(m I<\delta(\varepsilon)) \Rightarrow \sigma|I|<\varepsilon$.

The function $f$ is said to be $A C_{k}$ above (respectively below) on $E$ if $(\forall \varepsilon>0)(B \delta(\varepsilon)>0)(m I<\delta(\varepsilon)) \Rightarrow \sigma I<\varepsilon$ (respectively $\sigma I>-\varepsilon$ ).

Remark 1. Taking $k=1$ in the above definitions gives the corresponding definitions of $B V, A C, A C$ above (below) etcetera functions as in Ridder [14], Saks [19] and others.

The following theorem whose proof is omitted will be needed.

THEOREM 1. (see Theorem 2.3 and Coroilary 2.4 of De Sarkar and Das [10]). If $f$ is $A C_{k}$ above (respectively below) on $E$ and $D^{k-1} f$ exists on $E$, then $D^{k-1} f$ is $A C$ above (respectively below) on $E$. Notations and definitions not given here may be found in De Sarkar and Das [10].

2. $A C_{k}^{*}, B V_{k}^{*}, A C_{k}^{*} G$ functions.

For any two real numbers $c, d$ with $c<d$ define $\bar{S}_{k 1}(f ; c, d)=\sup \left\{Q_{k-1}\left(f ; x_{1}, x_{2}, \ldots, x_{k}\right)-Q_{k-1}\left(f ; c, x_{1}, \ldots, x_{k-1}\right)\right\}$ where the supremum is taken for all points $x_{i}, i=1,2, \ldots, k$, with $c<x_{1}<x_{2}<\ldots<x_{k} \leq d$; $\bar{S}_{k 2}(f ; c, d)=\sup \left\{Q_{k-1}\left(f ; x_{1}, x_{2}, \ldots, x_{k-1}, d\right)-Q_{k-1}\left(f ; x_{1}, x_{2}, \ldots, x_{k}\right)\right\}$ 
where the supremum is taken for all points $x_{i}, i=1,2, \ldots, k$, with $c \leq x_{1} \leq x_{2}<\ldots<x_{k}<d$.

similarly we define $\underline{S}_{k 1}(f ; c, d)$ and $\underline{S}_{k 2}(f ; c, d)$ by replacing the supremum by infimum in the above.

We now define

$$
S_{k 1}(f ; c, d)=\sup \left\{\left|Q_{k-1}\left(f ; x_{1}, x_{2}, \ldots, x_{k}\right)-Q_{k-1}\left(f ; c, x_{1}, \ldots, x_{k-1}\right)\right|\right\}
$$

where the supremum is taken for all points $x_{i}, i=1,2, \ldots, k$, with $c<x_{1}<x_{2}<\ldots<x_{k} \leq d$;

$$
s_{k 2}(f ; c, d)=\sup \left\{\left|Q_{k-1}\left(f ; x_{1}, x_{2}, \ldots, x_{k-1}, d\right)-Q_{k-1}\left(f ; x_{1}, x_{2}, \ldots, x_{k}\right)\right|\right\}
$$

where the supremum is taken for all points $x_{i}, i=1,2, \ldots, k$, with $c \leq x_{1}<x_{2}<\ldots<x_{k}<d$.

We take

$$
\begin{aligned}
& \bar{S}_{k}(f ; c, d)=\max \left\{\bar{S}_{k 1}(f ; c, d), \bar{S}_{k 2}(f ; c, d)\right\}, \\
& \underline{S}_{k}(f ; c, d)=\min \left\{\underline{S}_{k 1}(f ; c, d), \underline{S}_{k 2}(f ; c, d)\right\}
\end{aligned}
$$

and

$$
S_{k}(f ; c, d)=\max \left\{S_{k 1}(f ; c, d), S_{k 2}(f ; c, d)\right\} .
$$

It is easy to see that

$$
s_{k}(f ; c, d) \leq \bar{S}_{k}(f ; c, d)-\underline{s}_{k}(f ; c, d) .
$$

DEFINITION 1. The function $f$ is said to be $A C_{k}^{*}$ on $E$ if $D^{k-1} f(x)$ exists in an interval containing $E$ and if to each $\varepsilon>0$ there corresponds a $\delta>0$ such that for every finite sequence of nonoverlapping intervals $\left\{\left(c_{i}, d_{i}\right)\right\}$ whose end points belong to $E$ the inequality $\sum_{i}\left(d_{i}-c_{i}\right)<\delta$ implies $\sum_{i} s_{k}\left(f ; c_{i}, d_{i}\right)<\varepsilon$.

The function $f$ is said to be $A C_{k}^{*}$ above (respectively below), written $\overrightarrow{A C}_{k}^{*}$ (respectively $\underline{A C}_{k}^{*}$ ), on $E$ if $D^{k-1} f(x)$ exists in an interval containing $E$ and if for arbitrary $\varepsilon>0$, there exists a $\delta>0$ such that 


$$
\left.\sum_{i} \bar{S}_{k}\left(f ; c_{i}, d_{i}\right)<\varepsilon \quad \text { (respectively } \sum_{i} S_{k}\left(f ; c_{i}, d_{i}\right)>-\varepsilon\right)
$$

for every finite sequence of non-overlapping intervals $\left\{\left(c_{i}, d_{i}\right)\right\}$ whose end points are in $E$ and $\sum_{i}\left(d_{i}-c_{i}\right)<\delta$.

DEFINITION 2. We define

$$
\begin{aligned}
& \bar{V}_{k}^{*}(f ; E)=\sup \left\{\sum_{i} \bar{S}_{k}\left(f ; c_{i}, d_{i}\right)\right\}, \\
& V_{k}^{*}(f ; E)=\inf \left\{\sum_{i} S_{k}\left(f ; c_{i}, d_{i}\right)\right\}, \\
& V_{k}^{*}(f ; E)=\sup \left\{\sum_{i} S_{k}\left(f ; c_{i}, d_{i}\right)\right\}
\end{aligned}
$$

where the supremum and infimum are taken for all finite sequences of non-overlapping intervals $\left\{\left(c_{i}, d_{i}\right)\right\}$ whose end points are in $E$.

We have the following lemma whose proof is omitted.

LEMMA 1. $\bar{V}_{k}^{*}(f ; E) \geq 0 \geq \underline{V}_{k}^{*}(f ; E)$.

DEFINITION 3. If $V_{k}^{*}(f ; E)<+\infty$, then we say that $f$ is $B V_{k}^{*}$ on $E$. The function $f$ is said to be $B V_{k}$ above (respectively below), written $\bar{B} V_{k}^{*}$ (respectively $\underline{B} V_{k}^{*}$ ), on $E$ if $\bar{V}_{k}^{t}(f ; E)<+\infty$ (respectively $\left.V_{k}^{*}(f ; E)>-\infty\right)$.

Remark 2. Taking $k=1$ in Definitions $I$ and 3 gives the corresponding definitions of $A C^{*}, B V^{*}, A C^{*}$ above (below), $B V^{*}$ above (below) functions as in Ridder [14], Saks [19] and others.

We next prove the following theorems.

THEOREM 2. If $f$ is $A C_{k}^{*}$ on $E$, then $f$ is $A C_{k}$ on $E$.

Proof. Let $f$ be $A C_{k}^{*}$ on $E$ and let $\varepsilon>0$ be arbitrary. Let $I\left(x_{i, 1}, \ldots, x_{i, k-1}\right):\left(x_{i, 0}, x_{i, k}\right), i=1,2, \ldots, n$, be an elementary system in $E$. Since $f$ is $A C_{k}^{*}$ on $E$ there exists a $\delta>0$ such that (2.2) $\sum_{i=1}^{n} S_{k j}\left(f ; x_{i, 0}, x_{i, k}\right)<\varepsilon / 2 ; j=1,2$, for $\sum_{i=1}^{n}\left(x_{i, k}-x_{i, 0}\right)<\delta$.

In view of Theorem 3 of Russell [15], we have for $x_{i, 0}<y_{i}<x_{i, 1}$, $i=1,2, \ldots, n$, 


$$
\begin{aligned}
& \sum_{i=1}^{n}\left|Q_{k-1}\left(f ; x_{i, 0}, \ldots, x_{i, k-1}\right)-Q_{k-1}\left(f ; x_{i, 1}, \ldots, x_{i, k}\right)\right| \\
\leq & \sum_{i=1}^{n}\left|Q_{k-1}\left(f ; x_{i, 0}, y_{i}, x_{i, 1}, \ldots, x_{i, k-2}\right)-Q_{k-1}\left(f ; y_{i}, x_{i, 1}, \ldots, x_{i, k-1}\right)\right| \\
+ & \sum_{i=1}^{n}\left|Q_{k-1}\left(f ; y_{i}, x_{i, 1}, \ldots, x_{i, k-1}\right)-Q_{k-1}\left(f ; x_{i, 1}, \ldots, x_{i, k}\right)\right| \\
\leq & \sum_{i=1}^{n} S_{k 1}\left(f ; x_{i, 0}, x_{i, k}\right)+\sum_{i=1}^{n} S_{k 2}\left(f ; x_{i, 0}, \ldots, x_{i, k}\right) .
\end{aligned}
$$

Therefore using (2.2) we obtain

$$
\sum_{i=1}^{n}\left|Q_{k-1}\left(f ; x_{i, 0}, \ldots, x_{i, k-1}\right)-Q_{k-1}\left(f ; x_{i, 1}, \ldots, x_{i, k}\right)\right|<\varepsilon
$$

whenever $\sum_{i=1}^{n}\left(x_{i, k}-x_{i, 0^{\prime}}<\delta\right.$ and this proves the theorem.

Remark 3. It is easy to prove that if $f$ is $\overline{A C}_{k}^{*}$ (respectively $A C_{k}^{*}$ ) on $E$, then $f$ is $\overline{A C}_{k}$ (respectively $A C_{k}$ ) on $E$.

THEOREM 3. If $f$ is $B V_{k}^{*}$ on $E$, then $f$ is $B V_{k}$ on $E$.

Proof. Let $f$ be $B V_{k}^{*}$ on $E$. Then there exists a positive number $L$ such that for any finite sequence of non-overlapping intervals $\left\{\left(c_{i}, d_{i}\right)\right\}$ whose end points belong to $E$ we have

$$
\sum_{i} S_{k j}\left(f ; c_{i}, d_{i}\right)<L, j=1,2 .
$$

Let us consider any $\pi\left(x_{0}, x_{1}, \ldots, x_{n}\right)$ subdivision of $E$. We then have $k$ sets of non-overlapping intervals $\left(x_{i}, x_{i+k}\right), i \in A_{r}=\{r, k+r$, $2 k+r, \ldots \leq n\}$ and $r=0,1, \ldots, k-1$ so that

$$
\begin{aligned}
& \sum_{i=0}^{n-k}\left|Q_{k-1}\left(f ; x_{i}, x_{i+1}, \ldots, x_{i+k-1}\right)-Q_{k-1}\left(f ; x_{i+1}, \ldots, x_{i+k}\right)\right| \\
= & \sum_{i \in A_{r}}\left|Q_{k-1}\left(f ; x_{i}, x_{i+1}, \ldots, x_{i+k-1}\right)-Q_{k-1}\left(f ; x_{i+1}, \ldots, x_{i+k}\right)\right| .
\end{aligned}
$$

Let $x_{i}<y_{i}<x_{i+1}, i \in A_{p}$. Then in view of Theorem 3 of Russell [15] we have 


$$
\begin{aligned}
& \sum_{i=0}^{n-k}\left|Q_{k-1}\left(f ; x_{i}, x_{i+1}, \ldots, x_{i+k-1}\right)-Q_{k-1}\left(f ; x_{i+1}, \ldots, x_{i+k}\right)\right| \\
& \leq \sum_{i \in A_{r}}\left|Q_{k-1}\left(f ; x_{i}, y_{i}, x_{i+1}, \ldots, x_{i+k-2}\right)-Q_{k-1}\left(f ; y_{i}, x_{i+1}, \ldots, x_{i+k-1}\right)\right| \\
&+ \sum_{i \in A_{r}}\left|Q_{k-1}\left(f ; y_{i}, x_{i+1}, \ldots, x_{i+k-1}\right)-Q_{k-1}\left(f ; x_{i+1}, \ldots, x_{i+k}\right)\right| \\
& \leq \sum_{i \in A_{r}} S_{k 1}\left(f ; x_{i}, x_{i+k}\right)+\sum_{i \in A_{r}} S_{k 2}\left(f ; x_{i}, x_{i+k}\right) \\
&< 2 k L, \text { using }(2,3) . \\
& \text { Since } \pi \text { is an arbitrary subdivision of } E \text { it follows that } \\
& V_{k}(f ; E) \leq 2 k L \text { and hence } f \text { is } B V_{k} \text { on } E \text {. This completes the proof. }
\end{aligned}
$$

Remark 4. It can be proved easily that if $f$ is $\overline{B V}_{k}^{*}$ (respectively $\underline{B V_{k}^{*}}$ ) on $E$, then $f$ is $\overline{B V}_{k}$ (respectively $\underline{B V}_{k}$ ) on $E$.

THEOREM 4. If $f$ is $B V_{k}^{*}$ on $E$ and $D^{k-1} f$ exists on $(\alpha, \beta)$, then $f$ is $B V_{k}$ on $(\alpha, \beta)$.

Proof. Let $f$ be $B V_{k}^{*}$ on $E$ and suppose $D^{k-1} f$ exists on $(\alpha, \beta)$. Let $\pi\left(x_{0}, x_{1}, \ldots, x_{n}\right)$ be a subdivision of $(\alpha, \beta)$. Then

$$
\begin{aligned}
& \sum_{i=0}^{n-k}\left|Q_{k-1}\left(f ; x_{i}, \ldots, x_{i+k-1}\right)-Q_{k-1}\left(f ; x_{i+1}, \ldots, x_{i+k}\right)\right| \\
= & \sum_{i \in A_{r}}\left|Q_{k-1}\left(f ; x_{i}, \ldots, x_{i+k-1}\right)-Q_{k-1}\left(f ; x_{i+1}, \ldots, x_{i+k}\right)\right|
\end{aligned}
$$

where $A_{r}$ contains suffixes $r, k+r, 2 k+r, \ldots \leq n$ for $r=0,1, \ldots, k-1$.

Let $\varepsilon>0$ be arbitrary. Since $0^{k-1} f$ exists on $(\alpha, \beta)$ there exists a sequence of non-overlapping intervals $\left\{\left(y_{i}, y_{i+k}\right)\right\}, i \in A_{p}$, in $E$ such that

$$
\begin{aligned}
& \left|Q_{k-1}\left(f ; x_{i}, \ldots, x_{i+k-1}\right)-Q_{k-1}\left(f ; x_{i+1}, \ldots, x_{i+k}\right)\right| \\
& \leq S_{k}\left(y_{i}, y_{i+k}\right)+\varepsilon / k 2^{i} .
\end{aligned}
$$

This gives 


$$
V_{k}(f ;(\alpha, \beta)) \leq k V_{k}^{*}(f ; E)+\varepsilon / 2 .
$$

As $\varepsilon>0$ is arbitrary and $f$ is $B V_{k}^{*}$ on $E$, the theorem follows.

LEMMA 2. If $f$ is $B V_{k}^{*}$ on both $E_{1}=[a, c] \cap E$ and $E_{2}=[c, b] \cap E$ where $a<c<b$, and $(k-1)$ th divided differences are bounded on $(\alpha, \beta)$, then $f$ is $B V_{k}^{*}$ on $E$.

Proof. Suppose that $(k-1)$ th divided difference are bounded on $(\alpha, \beta)$ in absolute value by $K$, say. Consider any sequence of non-overlapping intervals $\left\{\left(c_{i}, d_{i}\right)\right\}, i=1,2, \ldots, n$, whose end points are in $E$ and let $c_{r}<c<d_{p}$ for some $r$. Then

$$
\begin{aligned}
& \sum_{i=1}^{n} S_{k}\left(f ; c_{i}, d_{i}\right)=\sum_{i=1}^{r-1} s_{k}\left(f ; c_{i}, d_{i}\right)+\sum_{i=r^{+}+1}^{n} S_{k}\left(f ; c_{i}, d_{i}\right)+S_{k}\left(f ; c_{p}, d_{p}\right) \\
& \leq V_{k}^{*}\left(f ; E_{1}\right)+V_{k}^{*}\left(f ; E_{2}\right)+2 K,
\end{aligned}
$$

and the lemma is proved.

THEOREM 5. If $f$ is $A C_{k}^{*}$ on $E$, then $f$ is $B V_{k}^{*}$ on $E$.

Proof. The proof follows using Definition 1, Definition 3 and Lerma 2.

Combining Theorems 4 and 5 we obtain.

COROLLARY 1. If $f$ is $A C_{k}^{*}$ on $E$, then $f$ is $B V_{k}$ on $(\alpha, \beta)$.

THEOREM 6. Let $c_{1}<d_{1} \leq c_{2}<d_{2} \leq \ldots \leq c_{k}<d_{k} \ldots$ be a set of points in $E$. If $D^{k-1} f$ exists on $(\alpha, \beta)$ and $f$ is $B V_{k}$ on $E$ then the series $\sum_{i} s_{k}\left(f ; c_{i}, d_{i}\right)$ is convergent.

Proof. Let $\left\{\left(p_{i}, q_{i}\right)\right\}, i=1,2, \ldots$, be a subsequence of all intervals $\left\{\left(c_{i}, d_{i}\right)\right\}, i=1,2, \ldots$, for each of which $\bar{s}_{k}\left(f_{i} p_{i}, q_{i}\right) \geq 0$. If $n$ is a positive integer, then

$$
\sum_{i=1}^{n} \bar{S}_{k}\left(f ; p_{i}, q_{i}\right) \leq v_{k}^{*}(f ; E)<+\infty
$$

and so 


$$
\sum_{i} \bar{s}_{k}\left(f ; p_{i}, q_{i}\right) \text { is convergent. }
$$

Next let $\left\{\left(r_{i}, t_{i}\right)\right\}, i=1,2, \ldots$, be a subsequence of $\left\{\left(c_{i}, d_{i}\right)\right\}$, $i=1,2, \ldots$, for each of which $\underline{S}_{k}\left(f ; r_{i}, t_{i}\right)<0$. For a fixed positive integer $n$ we consider the finite sequence $\left\{\left(r_{i}, t_{i}\right)\right\}, i=1,2, \ldots, n$. From the definition of $\underline{S}_{k}$ for each $\varepsilon>0$ arbitrary, there exists, for each $i(i=1,2, \ldots, n)$, a set of points $r_{i, 0}, r_{i, 1}, \ldots, r_{i, k}$ with $r_{i} \leq r_{i, 0}<r_{i, 1}<\ldots<r_{i, k} \leq t_{i}$ such that

$$
\begin{aligned}
& S_{k}\left(f ; r_{i}, t_{i}\right)+\varepsilon / 2^{i} \\
& >Q_{k-1}\left(f ; r_{i, 1}, \ldots, r_{i, k}\right)-Q_{k-1}\left(f ; r_{i, 0}, \ldots, r_{i, k-1}\right) .
\end{aligned}
$$

Thus we have an elementary system

$$
I\left(r_{i, 1}, \ldots, r_{i, k-1}\right):\left(r_{i, 0}, r_{i, k}\right), i=1,2, \ldots, n \text {, }
$$

in $(\alpha, \beta)$. Let $I_{c}$ denote the elementary system in $(\alpha, \beta) \cap\left[r, i, r_{n, k}\right]$ complementary to $I$. Then $I$ and $I_{c}$ together form an elementary system in $(\alpha, \beta)$ which we denote by

$$
J\left(n_{i+1}, \ldots, n_{i+k-1}\right):\left(n_{i}, n_{i+k}\right), i=0, k, 2 k,(m-1) k, m k
$$

where $n_{0}=r_{1,0}$ and $n_{(m+1) k}=r_{n, k}$. We consider $(k-1)$ elementary systems

$$
J_{s}\left(n_{i+1}, \ldots, n_{i+k-1}\right):\left(n_{i}, n_{i+k}\right),
$$

$i \in A_{s}=\{s, k+s, 2 k+s, \ldots \leq(m+1) k\}$, for each $s=1,2, \ldots,(k-1)$ so that

$$
\begin{aligned}
& \sigma I+\sigma I_{c}+\sigma J J_{1}+\ldots+\sigma J_{k-1} \\
= & Q_{k-1}\left(f ; n_{m k+1}, \ldots, n_{(m+1) k}\right)-Q_{k-1}\left(f ; n_{o}, n_{1}, \ldots, n_{k-1}\right) .
\end{aligned}
$$

In view of Theorem 4 above and Theorem 4 of Russell [15], the $(k-1)$ th divided differences of $f$ are bounded in $(\alpha, \beta)$.

Therefore $a I \geq-\varepsilon K-k V_{k}(f ;(\alpha, \beta))$ where $K$ is the bound of the $(k-1)$ th 
divided differences on $(\alpha, \beta)$. Thus from (2.4) it follows that

$$
\sum_{i=1}^{n} \underline{S}_{k}\left(f ; r_{i}, t_{i}\right)>\sigma I \geq-2 K-k v_{k}(f ;(\alpha, \beta)) \text {. }
$$

Since, by Corollary $1, f$ is $B V_{k}$ on $(\alpha, \beta)$ and $n$ is arbitrary, the series $\sum_{i} S_{k}\left(f ; r_{i}, t_{i}\right)$ is convergent. Because, by (2.1)

$$
\sum_{i} s_{k}\left(f ; c_{i}, d_{i}\right) \leq \sum_{i} \bar{S}_{k}\left(f ; p_{i}, q_{i}\right)-\sum_{i} S_{k}\left(f ; r_{i}, t_{i}\right)
$$

the theorem follows.

COROLLARY 2. Let $E$ be a bounded closed set with contiguous intervals $\left(a_{n}, b_{n}\right), n=1,2, \ldots$. . If $f$ is $A C_{k}^{*}$ on $E$, then the series $\sum_{n} s_{k}\left(f ; a_{n}, b{ }_{n}\right)$ converges.

THEOREM 7. If $f$ is $A C_{k}$ on $E \subseteq[a, b]$, then there exists $a$ fronction $F$ in $[a, b]$ such that

(i) $F$ is $A C_{k}^{*}$ on $E$,

(ii) $F^{(k-1)}$ is $A C^{*}$ on $E$,

(iii) $F(x)=f(x)$ for $x \in E$,

(iv) $F^{(r)}(x)=D^{r} f(x), r=1,2, \ldots,(k-1)$ on $E$.

Proof. Let $f$ be $A C_{k}$ on $E \subseteq[a, b]$. Then Theorem 3.3 of De Sarkar and Das [10] yields a function $F$ in $[a, b]$ such that $F(x)=f(x)$ for $x \in E$ (which proves (iii)) and $F$ is $A C_{k}$ on $[a, b]$. By Lemma 1 and the subsequent Note of Das and Lahiri [7] $D^{k-1} F$ exists on $[a, b]$. Again using Theorem 1 of Das and Lahiri [7] and Theorem 4 of Russell [15] we see that the $(k-1)$ th divided differences of $F$ are bounded in $[a, b]$. consequently $b^{k-1} F$ is bounded in $[a, b]$ and so, by Theorem 3 of Oliver [13], $D^{k-1} F(x)=F_{k-1}(x)=F^{(k-1)}(x)$ for all $x$ in $[a, b]$. Then by Theorem 1 it follows that $F^{(k-1)}$ is $A C$ on $[a, b]$. Hence (i) and (ii) follow from above and the fact that a function which is $A C_{k}$ (respectively $A C$ ) on $[a, b]$ is necessarily $A C_{k}^{*}$ (respectively 
$A C^{*}$ ) on any subset of $[a, b]$. It is easy to see that (iv) follows from Lemma 1 and the subsequent Note of Das and Lahiri [7] .

This completes the proof.

THEOREM 8. If $f$ is $A C_{k}^{*}$ on $E$, then $f$ is $A C_{k}^{*}$ on the closure $\bar{E}$ of $E$.

Proof. Since $D^{k-1} f$ exists on $E$ we have, for $c \in \bar{E}$ and $\lambda \in E$, $S_{k 1}(f ; c, \lambda) \rightarrow 0$ and $S_{k 2}(f ; c, \lambda) \rightarrow 0$ as $\lambda \rightarrow c$ over the points of $E$.

It then follows that $\left.\left.Q_{k-1}\left(f ; y_{1}, y_{2}, \ldots, y_{k-1}, \lambda\right)+Q_{k-1}\right) f ; x_{1}, x_{2}, \ldots, x_{k-1}, c\right)$

as $\lambda \rightarrow c, x_{i}, y_{i} \in[a, b], c<x_{i} \leq y_{i}<\lambda$ or $c>x_{i} \geq y_{i}>\lambda$

according as $\lambda>c$ or $\lambda<c$. Now if $d \in \bar{E}$ and $\mu \in E$, then it is not difficult to show that

$$
\begin{aligned}
& \qquad\left|Q_{k-1}\left(f ; x_{1}, x_{2}, \ldots, x_{k}\right)-Q_{k-1}\left(f ; c, x_{1}, x_{2}, \ldots ; x_{k-1}\right)\right| \\
& =\lim _{\lambda \rightarrow c}\left|Q_{k-1}\left(f ; y_{1}, y_{2}, \ldots, y_{k}\right)-Q_{k-1}\left(f ; \lambda, y_{1}, y_{2}, \ldots, y_{k-1}\right)\right|, \\
& x_{i}, y_{i} \in[a, b], c<x_{1}<\ldots<x_{k} \leq d, \lambda<y_{1}<y_{2}<\ldots<y_{k} \leq \mu, x_{i} \geq y_{i} \\
& \text { or } x_{i} \leq y_{i}(i=1,2, \ldots, k) \text { according as } \lambda>c \text { or } \lambda<c . \text { This shows } \\
& \text { that }
\end{aligned}
$$

$$
s_{k 1}(f ; c, d) \leq \lim _{\lambda \rightarrow c, \mu \rightarrow d} \inf _{k 1}(f ; \lambda, \mu) .
$$

Similarly it can be shown that

$$
S_{k 2}(f ; c, d) \leq \lim _{\lambda \rightarrow c,} \inf _{\mu \rightarrow d} S_{k 2}(f ; \lambda, \mu)
$$

and hence

$$
S_{k}(f ; c, d) \leq \lim _{\lambda \rightarrow c,} \inf _{\mu \rightarrow d} S_{k}(f ; \lambda, \mu)
$$

The theorem now follows easily.

THEOREM 9. If $f$ is $A C_{k}^{*}$ on a closed set $E \subseteq[a, b]$, then $D^{k} f$ exists and equals $\left(D^{k-1} f\right)^{\prime}$ ap almost everywhere in $E$.

Proof. Let $f$ be $A C_{k}^{*}$ on $E$. We set $g(x)=f(x)-F(x)$ where $F(x)$ is the function obtained by applying Theorem 7 on $E$. Then 


$$
\vec{D}^{r} g(x)=0 \text { for } x \in E, r=0,1,2, \ldots,(k-1) .
$$

By Theorem $6, g$ is $A C_{k}^{*}$. on $E$. Let $\left\{\left(c_{i}, d_{i}\right)\right\}$ be the set of complementary intervals of $E$ in $[a, b]$. Define

$$
\begin{aligned}
h(x) & =0 \quad \text { for } x \in E \\
& =s_{k}\left(g ; c_{1}, d_{i}\right) \text { for } c_{i}<x<d_{i} .
\end{aligned}
$$

since $g$ is $A C_{k}^{*}$ on $E$ it follows that $h$ is $A C^{*}$ on $E$. Also $F^{(k-1)}$ is $A C^{*}$ on $E$. Hence $h^{1}$ and $F^{(k)}$ exist almost everywhere in $E$. Since $D^{k-1} f=F^{(k-1)}$ on $E$ it follows that

$$
F^{(k)}(x)=\left(D^{k-1}\right){ }_{\alpha q}^{\prime}(x)
$$

holds whenever $x$ is a point of density of $E$. It can be shown that $D^{k} f(n)=F^{(k)}(n)$ holds whenever $n$ is a point of density of the set where $F^{(k)}$ and $h^{1}$ exist finitely. This completes the proof. COROLLARY 3. If $f$ is $A C_{k}^{*}$ on the closed set $E \subset[a, b]$, then $D^{k} f$ is Lebesgue integrable on $E$.

DEFINITION 4. The function $f$ is said to be $A C_{k}^{*} G$ below (respectively above) written $\underline{A C}_{k}^{*} G$ (respectively $\overline{A C}_{k}^{*} G$ ), on $E$ if $E$ can be expressed as a countable union of sets on each of which $f$ is $A C_{k}^{*}$ (respectively $\overline{A C}_{k}^{*}$ ). The function $f$ is $A C_{k}^{*} G$ on $E$ if $E$ is a countable union of sets on each of which $f$ is $A C_{k}^{*}$.

we shall use the notation $\left[A C_{k}^{*} G\right]$ etcetera instead of $A C_{k}^{*} G$ etcetera to mean that the set $E$ can be expressed as a countable union of closed sets on each of which $f$ is $A C_{k}^{*}$ etcetera.

We note that taking $k=1$ in the above definition gives the corresponding definitions of $A C^{*} G, A C^{*} G$ above (below), [AC* $\left.G\right]$ above (below) etcetera functions as in Ridder [14], Saks [19] and others.

In view of Theorem 8 we have the following remark.

Remark 5. If $f$ is $A C_{k}^{*} G$ on $E \subset[a, b]$, then $f$ is $\left[A C_{k}^{*} G\right]$ on $\bar{E}$, the closure of $E$. 
THEOREM 10. If $\bar{D}^{k} f(x)<+\infty$ on $E$, then $f$ is $\overline{A C}_{k}^{*} G$ on $E$.

Proof. For a fixed positive integer $r$ let $E_{r}$ denote the set of all points $x$ of $E$ such that for every $x_{1}, x_{2}, \ldots, x_{k}$,

$$
0<\left|x_{1}-x\right|<\ldots<\left|x_{k-1}-x\right|<\left|x_{k}-x\right| \leq 1 / r
$$

implies

$$
k ! Q_{k}\left(f ; x, x_{1}, \ldots, x_{k-1}, x_{k}\right)<r .
$$

Let $E_{r i}=E_{r} \cap[i / r,(i+1) / r]$. Then

$$
E=\stackrel{\infty}{u}=1_{i=-\infty}^{\infty} E_{r i}
$$

We shall show that $f$ is $\overline{A C}_{k}^{*}$ on each $E_{m i}$. Let $\alpha_{1}, \alpha_{2} \in E_{m i}$ with $\alpha_{1}<x_{1}<\ldots<x_{k}<\alpha_{2}$. Then from (2.5), using Lemma 4 of Russell [15] we get

$Q_{k-1}\left(f ; x_{1}, \ldots, x_{k}\right)-Q_{k-1}\left(f ; \alpha_{1}, x_{1}, \ldots, x_{k-1}\right)<\frac{r}{k !}\left(x_{k}-\alpha_{1}\right)<\frac{r}{k !}\left(\alpha_{2}-\alpha_{1}\right)$

and

$Q_{k-1}\left(f ; x_{1}, \ldots, x_{k-1}, \alpha_{2}\right)-Q_{k-1}\left(f ; x_{1}, \ldots, x_{k}\right)<\frac{r}{k !}\left(\alpha_{2}-x_{k}\right)<\frac{r}{k !}\left(\alpha_{2}-\alpha_{1}\right)$.

Thus it follows that $\bar{S}_{k j}\left(f ; \alpha_{1}, \alpha_{2}\right)<\frac{r}{k ! l}\left(\alpha_{2}-\alpha_{1}\right), j=1,2$, and therefore $\bar{S}_{k}\left(f ; \alpha_{1}, \alpha_{2}\right)<\frac{r}{k !}\left(\alpha_{2}-\alpha_{1}\right)$. It is now easy to prove that $f$ is $\overline{A C}_{k}^{*}$ on each $E_{r i}$. The proof is completed by $(2.6)$.

In a similar way it can be shown that if $\underline{D}^{k} f>-\infty$, then $f$ is $A C_{k}^{*} G$ on $E$. Hence we get the following corollary.

COROLLARY 4. If $-\infty<\underline{D}^{k} f(x) \leq \vec{D}^{k} f(x)<+\infty$ on $E$, then $f$ is $A C_{k}^{*} G$ on $E$.

THEOREM 11. Let $f$ be $\left[A C_{k}^{*} G\right]$ below on $[a, b]$ and let $\underline{D}^{k} f \geq 0$ almost everywhere in $[a, b]$. If $P$ is any perfect set in $[a, b]$ such that $f$ is $k$-convex on the complementary intervals of $P$, then there is an interval $[2, m]$ containing the points of $P$ such that $f$ is $k$-convex on $[l, m]$. 
Proof. Since $f$ is $\left[A C_{k}^{*} G\right]$ below on $[a, b]$ there is a sequence of closed sets $\left\{E_{i}\right\}$ such that $[a, b]=y_{i} E_{i}$ and $f$ is $A C_{k}^{*}$ below on each $E_{i}$. Then $P=\frac{y}{2}\left(P \cap E_{i}\right)$. So by Baire's Theorem, (Saks [19,p 54]) there exists $i_{0}$ and an interval $[l, m]$ such that $P \cap[l, m] \subset P \cap E_{i_{0}}$. Hence $f$ is $A C_{k}^{*}$ below on $P \cap[Z, m]$. Therefore, by Remark $2, f$ is $A C_{k}$ below on $P \cap[l, m]$ and hence, by Theorem $1, D^{k-1} f$ is $A C$ below on $P \cap[Z, m]$. Since $f$ is $k$-convex on the complementary intervals of $P$ and $D^{k-1} f$ is a Darboux function it follows that $D^{k-1} f$ is continuous and non-decreasing on the closure of each complementary intervals of $P$. Hence $D^{k-1} f$ is $A C$ below on $[l, m]$. Therefore $D^{k-1} f$ is nondecreasing on $[l, m]$. This implies, in view of Bullen [1, Corollary 8] and oliver [13, Theorem 3] (also repeated in verblunsky [21, Theorem 1 (ii)]), that $f$ is $k$-convex on $[l, m]$. This completes the proof. THEOREM 12. Let $f$ be $\left[A C_{k}^{*} G\right]$ be zow on $[a, b]$. If $\underline{D}^{k} f \geq 0$ almost everywhere in $[a, b]$, then $f$ is $k$-convex on $[a, b]$.

Proof. Let $G$ be the set of all points $x$ in $[a, b]$ such that there is a neighbourhood $N_{x}=\left(\alpha_{x}{ }^{8}{ }_{x}\right)$ of $x$ in which $f$ is $k$-convex. Then the set $H=[a, b] \backslash G$ is perfect. If possible suppose that $H$ is non-empty. Let $\left\{\left(a_{i}, b_{i}\right)\right\}$ be the set of complementary intervals of $H$ in $[a, b]$. Then $f$ is $k$-convex in each interval $\left(a_{i}, b_{i}\right)$. Thus, by Theorem 11, there is an interval $[l, m]$ containing points of $H$ such that $f$ is $k$-convex on $[l, m]$. This contradicts the fact that $[l, m]$ contains points of $H$. Therefore $H$ is empty.

Let $N_{x}$ be the neighbourhood of $x$ in which $f$ is $k$-convex. By The Heine-Borel Theorem there is a finite subcover $N_{x}^{(j)}, j=1,2, \ldots, n$, of $[a, b]$. Since the neighbourhoods are not disjoint, by the application of Theorem 2 of Russell [15] it is easy to show that $f$ is $k$-convex on $[a, b]$ and thus the theorem is proved.

COROLLARY 5. If $f$ is $\left[A C_{k}^{*} G\right]$ on $[a, b]$ and if one of $\vec{D}^{k} f, \underline{D}^{k} f$ 
vanishes almost everywhere in $[a, b]$, then $f$ is a polynomial of degree $(k-1)$ at most.

THEOREM 13. If $f$ is $\left[A C_{k}^{*} G\right]$ on $E \subset[a, b]$, then for almost all $x$ in $E, D^{k} f(x)$ and $\left(D^{k-1} f\right)_{\alpha p}^{\prime}(x)$ exist and are equar.

Proof. The proof follows directly from the definition of $\left[A C_{k}^{*} G\right]$ and Theorem 9.

3. The $p^{k}-, D^{k}-, P^{k}-$ integrals.

DEFINITION 5. Let $f$ be a real valued function defined on $[a, b]$. Then a function $M$ continuous on $[a, b]$ is called a $P^{k}$-major function (or simply a major function) of $f$ on $[a, b]$ if and only if

(i) $D^{n} M$ exists and is finite on $[a, b], 1 \leq r \leq k-1$;

(ii) $\underline{D}^{k} M\left(x ; \geq f(x), x \in[a, b] \backslash E_{1}, m E_{1}=0\right.$;

(iii) $\underline{D}^{k} M(x)>-\infty, x \in[a, b] \backslash C, C$ is countable;

(iv) $D^{r} M(a)=0,0 \leq r \leq k-1$.

If $-m$ is a major function of $-f$, then $m$ is called a minor function or more precisely a $P^{k}$-minor function of $f$ on $[a, b]$.

It is clear from the definition that $f$ need only be finite or indeed defined almost everywhere.

For $a<c \leq b$ define $\bar{F}(c)$, the upper $p^{k}$-integral of $f$ on $[a, b]$, by $\bar{F}(c)=\vec{p}^{k}-\int_{a}^{c} f=\inf \{t: t=M(c), M$ is a major function of $f\}$. In a similar manner the lower $p^{k}$-integral $\underline{F}(c)=\underline{p}^{k}-\int_{a}^{c} f$ is defined. If $\vec{E}(c)=\underline{F}(c)$ we write the common value $F(c)=p^{k}-\int_{a}^{c} f$ and if further this value is finite we say $f$ is $P^{k}$-integrable on $[a, c]$.

Remark 6. Bullen and Mulkhopadhyay [4, Theorem 4.4] shows that if the $(k-1)$ th Peano derivative $f_{k-1}$ and the $(k-1)$ th Riemann derivative $D^{k-1} f$ exist at $x$, then the one sided upper (respectivly lower) $k$ th 
Peano derivative and one sided upper (respectively lower) $k$ th Riemann* derivative at $x$ are equal. Thus it follows that the above integral is same as the $P^{n}$-integral, for $n=k$, defined in Bullen [2].

Standard arguments (see Bullen [2]) will prove the following results.

THEOREM 14. If $f$ is $P^{k}$-integrable, then

(i) for every major function $M$ and every minor function $m, M-F$ and $F-m$ are $r$-convex on $[a, b], 0 \leq r \leq k$,

(ii) $D^{k}$ exists on $(a, b)$;

(iii) $\quad F(a)=D^{r} F(a)=0,1 \leq r \leq k-1$;

(iv) $D^{2} F(b)$ exists, $1 \leq r \leq k-1$.

THEOREM 15. If $f$ is $P^{k}$-integrable on $[a, c]$ and on $[c, b]$, then $f$ is $P^{k}$-integrable on $[a, b]$.

THEOREM 16. Suppose $f$ is $P^{k}$-integrable on every $[c, d]$, $a<c<d<b$ and put $I(c, d)=P^{k}-\int_{c}^{d} f$. Suppose further that

$$
\lim _{c \rightarrow a} \frac{I(c, d)}{(c-a)^{k-1}}=0 \text {, and }
$$

(ii) there is a polynomial $P$ of degree (k-1) at most such that

$$
\lim _{d \rightarrow b} \frac{I(c, d)-p(d)}{(b-d)^{k-1}}=0
$$

then $f$ is $p^{k}$-integrable on $[a, b]$ and

$$
P^{k}-\int_{a}^{b} f=\lim _{\substack{c \rightarrow a \\ d \rightarrow b}} I(c, d)
$$

THEOREM 17. If $f$ is $P^{k}$-integrable on $[a, b]$ and $F$ is its $P^{k}$-integral, then $D^{k_{F}}$ exists and equals $f$ almost everywhere.

THEOREM 18. If $E$ is a closed bounded set with end points $\alpha^{\prime}$ and $\beta^{\prime}$ and contiguous intervals $\left(a_{n}, b_{n^{\prime}}{ }^{\prime}\right.$ in $\left(\alpha^{\prime}, \beta^{\prime}\right), n=1,2, \ldots$, and if 
(i) $f$ is Lebesgue integrable on $E$,

(ii) $f$ is $P^{k}$-integrable on each $\left[a_{n}, b_{n}\right], n=1,2, \ldots$,

(iii) $\sum_{n} S_{k}\left(F(n) ; a_{n}, b_{n}\right)<+\infty$,

where $F(n)$ is the $P^{k}$-integral of $f$ on $\left[a_{n}, b_{n}\right]$, then $f$ is $P^{k}$ integrable on $\left[\alpha^{\prime}, \beta^{\prime}\right]$.

DEFINITION 6 . The function $f$ is said to be $D^{k}$-integrable on $[a, b]$ if and only if there is a function $F$ such that

(i) $F$ is $A C_{k}^{*} G$ on $[a, b]$,

(ii) $D^{r} F(a)=0,0 \leq r \leq k-1$,

(iii) $D^{k} F(x)=f(x)$ almost everywhere.

Further we call $F$ the indefinite $D^{k}$-integral of $f$ and write $F(x)=D^{k}-\int_{a}^{x} f(x), a<x \leq b$.

It follows from Remark 5 and Corollary 5 that if such an $F$ exists it is unique.

We shall now show that the $P^{k}$ - and $D^{k}$-integrals are completely equivalent.

THEOREM 19. If $f$ is $P^{k}$-integrable on $[a, b]$, then it is $D^{k}$ integrable on $[a, b]$ to the same value and conversely.

Proof. (a) Let $f$ be $P^{k}$-integrable on $[a, b]$ and $F$ be its $P^{k}$-integral on $[a, x], a<x \leq b$. Let $\varepsilon>0$ be arbitrary. There is a major function $M$ such that

$$
0 \leq D^{k-1} R=D^{k-1}(M-F) \leq(k-1) ! \varepsilon / 2 \text {, }
$$

where $R=M-F$. By Theorem 10, $M$ is $A C_{k}^{*} G$ below on $[a, b]$. Therefore there exists a sequence of sets $\left\{E_{j}\right\}$ such that $[a, b]=y_{j} E_{j}$ and $M$ is $A C_{k}^{*}$ below on each $E_{j}$. There is a $\delta>0$ such that if $\left\{\left(c_{i}, d_{i}\right)\right\}$, $i=1,2, \ldots, n$, is any sequence of non-overlapping intervals with end 
points in $E_{j}$ and $\sum_{i=1}^{n}\left(d_{i}-c_{i}\right)<\delta$, then

$$
\sum_{i=1}^{n} S_{k}\left(M ; c_{i}, d_{i}\right)>-\varepsilon / 2
$$

For any set of points $x_{i, r}, r=1,2, \ldots, k$, with $c_{i}<x_{i, 1}<x_{i, 2}<\ldots$ $<x_{i, k} \leq d_{i}$ we have

$$
\begin{aligned}
& Q_{k-1}\left(F ; x_{i, 1}, x_{i, 2}, \ldots, x_{i, k}\right)-Q_{k-1}\left(F ; c_{i}, x_{i, 1}, \ldots, x_{i, k-1}\right) \\
& =\left\{Q_{k-1}\left(M: x_{i, 1}, x_{i, 2}, \ldots, x_{i, k}\right)-Q_{k-1}\left(M ; c_{i}, x_{i, 1}, \ldots, x_{i, k-1}\right)\right\} \\
& -\left\{Q_{k-1}\left(R ; x_{i, 1}, x_{i, 2}, \ldots, x_{i, k}\right)-Q_{k-1}\left(R ; c_{i}, x_{i, 1}, \ldots, x_{i, k-1}\right)\right\} .
\end{aligned}
$$

Since by Theorem 14, $R$ is $k$-convex on $[a, b]$ it is easy to deduce from this that

$$
\begin{aligned}
& Q_{k-1}\left(F ; x_{i, 1}, x_{i, 2}, \ldots, x_{i, k}\right)-Q_{k-1}\left(F ; c_{i}, x_{i, 1}, \ldots, x_{i, k-1}\right) \\
& \geq Q_{k-1}\left(M ; x_{i, 1}, \ldots, x_{i, k}\right)-Q_{k-1}\left(M ; c_{i}, x_{i, 1}, \ldots, x_{i, k-1}\right) \\
& -\left(D^{k-1}{ }_{R\left(d_{i}\right)}-D^{k-1} R\left(c_{i}\right)\right\} /(k-1) ! .
\end{aligned}
$$

Hence, since $D^{k-1} R$ is non-decreasing, we get

$$
\sum_{i=1}^{n} \underline{S}_{k 1}\left(F ; c_{i}, d_{i}\right) \geq \sum_{i=1}^{n} \underline{S}_{k}\left(M ; c_{i}, d_{i}\right)-\left\{D^{k-1} R(b)-D^{k-1} R(a)\right\} /(k-1) !
$$

and so

$$
\sum_{i=1}^{n} S_{k 1}\left(E ; c_{i}, d_{i}\right)>-\varepsilon / 2-\varepsilon / 2=-\varepsilon \text { whenever } \sum_{i=1}^{n}\left(d_{i}-c_{i}\right)<\delta .
$$

In a similar way it can be shown that

$$
\sum_{i=1}^{n} \underline{S}_{k 2}\left(E ; c_{i}, d_{i}\right)>-\varepsilon \text { whenever } \sum_{i=1}^{n}\left(d_{i}-c_{i}\right)<\delta \text { and therefore } F
$$

is $A C_{k}^{*}$ below on each $E_{j}$ and hence $A C_{k}^{*} G$ below on $[a, b]$.

$$
\text { However, since }-f \text { is also } P^{k} \text {-integrable, } F \text { is also } A C_{k}^{*} G
$$

above on $[a, b]$ and hence $F$ is $A C_{k}^{*} G$ on $[a, b]$.

This and Theorems 14 and 17 above show that $f$ is $D^{k}$-integrable on $[a, b]$ and that 


$$
D^{k}-\int_{a}^{x} f=P^{k}-\int_{a}^{x} f, a \leq x \leq b .
$$

(b) Suppose $f$ is $D^{k}$-integrable on $[a, b]$ and let

$E_{1}=\left\{x: f\right.$ is not $P^{k}$-integrable in any neighbourhood of $\left.x\right\}$.

Then, clearly, $E_{1}$ is closed and let $\left\{\left(a_{n}, b_{n}\right)\right\}$ denote the intervals of the complement of $E_{1}$ in $[a, b]$.

$$
\text { If } a_{n}<\alpha^{\prime}<\beta^{\prime}<b_{n} \text {, then } f \text { is } p^{k} \text {-integrable on }\left[\alpha^{\prime}, \beta^{\prime}\right] \text {. }
$$

If $F$ is the indefinite $D^{k}$-integral of $f$ on $[a, b]$, then since from the definition of $D^{k}$-integral it is clear that

$F(x)-\sum_{m=0}^{k-1} \frac{\left(x-\alpha^{\prime}\right)^{m}}{m !} D^{m} F\left(\alpha^{\prime}\right)$, where $D^{\circ} F\left(\alpha^{\prime}\right)=F\left(\alpha^{\prime}\right)$,

is the indefinite $D^{k}$-integral of $f$ on $\left[\alpha^{\prime}, B^{\prime}\right]$ we have from (a) that

$$
P^{k}-\int_{\alpha^{\prime}}^{\beta^{\prime}} f=F\left(\beta^{\prime}\right)-\sum_{m=0}^{k-1} \frac{\left(\beta^{\prime}-\alpha^{\prime}\right)^{m}}{m !} D^{m} F\left(\alpha^{\prime}\right) \text {. }
$$

Since the right hand side of (3.1) satisfies the conditions of Theorem 16 on $\left[a_{n}, b_{n}\right]$ we have that $f$ is $p^{k}$-integrable on $\left[a_{n}, b_{n}\right]$ and

$$
P^{k}-\int_{a_{n}}^{b} f=F\left(b_{n}\right)-\sum_{m=0}^{k-1} \frac{\left(b_{n}-a_{n}\right)^{m}}{m !} D^{m} F\left(a_{n}\right)
$$

Hence, by Theorem 15, $E_{1}$ is a perfect set.

If possible, let $E_{1}$ be non-empty. Since $F$ is $\left[A C_{k}^{*} G\right]$ on $[a, b]$ by Baire's Theorem it follows that $E_{1}$ contains a portion $Q$ such that $F$ is $A C_{k}^{*}$ on $\bar{Q}$, the closure of $Q$. Let $c, d$ be the end points of $\bar{Q}$ and $\left(c_{n}, d_{n}\right), n=1,2, \ldots$, be the contiguous intervals of $\bar{Q}$ in $[c, d]$. Then by Corollary 2 we have $\sum_{n} s_{k}\left(F ; c_{n}, d_{n}\right)<+\infty$. Also by Corollary 3, $D^{k_{F}}$ is Lebesgue integrable on $\bar{Q}$. Hence, by Theorem 18 , $f$ is $P^{k}$-integrable on $[c, d]$. 
This contradiction shows that $E_{1}$ is empty and thus the proof of the theorem is complete.

DEFINITION 7. Let $f$ be a finite real valued function on $[a, b]$. Then a continuous function $M$ is called a $p^{k}$-major function of $f$ if and only if

(i) $D^{r} M$ exists and is finite, $1 \leq r \leq k-1$;

(ii) $\underline{D}^{k} M(x) \geq f(x)$ for almost all $x$;

(iii) $M$ is $\left[A C_{k}^{*} G\right]$ below on $[a, b]$;

(iv) $D^{r} M(a)=0,0 \leq r \leq k-1$.

In a similar manner $p^{k}$-minor functions can be defined. Then by a standard procedure (see Bullen [2], James [12]), this leads to a definition of an integral of Perron type, the $p^{k}$-integral, say.

It follows from the above definition that the function $f$ need only be finite, or indeed defined, almost everywhere in $[a, b]$.

We shall show, Theorem 20 below, that the $p^{k}$-integral is equivalent to the $P^{k}$-integral (and consequently to the $D^{k}$-integral). To show this we require the following results.

LEMMA 3. If $M$ is any $p^{k}$-major function of $f$, and $m$ is any $p^{k}$-minor function of $f$, then $M-m$ is k-convex.

Proof. The proof follows immediately from Theorem 12 .

COROLLARY 6. If $f$ is $p^{k}$-integrable, and $M$ and $m$ are as in Lemma 3 , then $M-F$ and $F-m$ are $k$-convex, where $F$ is the indefinite $p^{k}$-integral of $f$.

COROLLARY 7. If $f$ is $p^{k}$-integrable on $[a, b]^{\circ}$, and $F$ is its indefinite $P^{k}$-integral, then $D^{r} F$ exists, $a \leq x \leq b, 1 \leq r \leq k-1$.

LEMMA 4. If $f$ is $p^{k}$-integrable on $[a, b]$ with zero as its indefinite $p^{k}$-integral, then $f(x)=0$ for almost all $x$ in $[a, b]$. 
Proof. Suppose that $f>0$ on some set of positive measure. Then for some $\alpha^{\prime}, \beta^{\prime}, a<\alpha^{\prime}<\beta^{\prime}<b$ and some $s>0, \varepsilon>0$ there is a set $A \subset\left[\alpha^{\prime}, B^{\prime}\right]$ such that $m A>\varepsilon$ and $x \in A$ implies $f(x)>s$.

If $M$ is any $p^{k}$-major function of $f$, then by corollary $6, M$ is $k$-convex. Let $B=\left\{x: M^{(k)}(x) \geq s\right\}$; then by (ii) in Definition 7 and Bullen and Mukhopadhyay [4, Theorem 4.4] it follows that $A \subset B$ and so $m B>\varepsilon$.

Again since $M$ is $k$-convex, by Bullen [1, Theorem 12], we have

$$
m B \leq \frac{2 k}{s}\left\{M^{(k-1)}\left(B^{\prime}\right)-M^{(k-1)}\left(\alpha^{\prime}\right)\right\} .
$$

But $M$ can be chosen so that $M\left(\beta^{\prime}\right)$ is arbitrarily small and so by Bullen [1, Corollary $8(\mathrm{~b})]$ the right hand side of (3.3) can, by the correct choice of $M$, be made less than $\varepsilon$. This contradicts (3.2) and so $f(x) \leq 0$ almost everywhere. In a similar manner it can be shown that $f(x) \geq 0$ almost everywhere, which completes the proof.

COROLLARY 8 . If $f$ and $g$ are both $p^{k}$-integrable with the scome indefinite $p^{k}$-integrals, then $f(x)=g(x)$ for almost all $x$.

THEOREM 20. The $p^{k}$-integral is equivalent to the $p^{k}$-integral.

Proof. (a) Let $f$ be $P^{k}$-integrable. Then by Theorem 19, $f$ is $D^{k}$-integrable. Therefore there is a function $F$ such that $F$ is $A C_{k}^{*} G$ on $[a, b], D^{r} F(a)=0,0 \leq r \leq k-1$ and $D^{k} f(x)=f(x)$ for almost all of $x$. Then, by Remark $5, F$ is $\left[A C_{k}^{*} G\right]$ on $[a, b]$ and so is both a $p^{k}$-major and a $p^{k}$-minor function of $f$. Hence $f$ is $p^{k}$-integrable with $F$ as its indefinite $P^{k}$-integral; but by Theorem 19, $F$ is also the indefinite $P^{k}$-integral of $f$.

(b) Let $f$ be $p^{k}$-integrable with indefinite $p^{k}$-integral $F$. Proceeding as in (a) of the proof of Theorem 19 it follows that $F$ is $\left[A C_{k}^{*} G\right]$ on $[a, b]$. By Theorem 13, $D^{k} E$ exists almost everywhere and so from Definition $7, D^{k} F$ is $D^{k}$-integrable with $F$ as its indefinite 
$D^{k}$-integral. By part (a) above then $D^{k} F$ is $p^{k}$-integrable with $F$ as its indefinite $p^{k}$-integral. Hence by Corollary $8, D^{k} E=f$ almost everywhere, that is to say, $f$ is $D^{k}$-integrable and so $P^{k}$-integrable with $F$ as its indefinite $P^{k}$-integral. This completes the proof.

Remark 7. From Theorem 20 and Remark 6 it follows that the $P^{k}$-, $D^{k}-, P^{k}$-integrals are equivalent to the $P^{k}-, D^{k}$ - integrals in Bullen [2] and also to the $p^{k}$-integral in Bullen and Mukhopadhyay [3]. It therefore follows, in view of Bullen [2, Theorem 16], that $f$ is $p^{k}$ integrable on $[a, b]$ if and only if it is $C_{k-1} P$-integrable in $[a, b]$. If $F$ is the $P^{k}$-integral of $f$ then

$$
\begin{aligned}
& D^{k-1} F(x)=c_{k-1} P-\int_{a}^{x} f, \\
& F(x)=P-\int_{a}^{x} c_{1} P-\int_{a}^{x} c_{2} P-\int_{a}^{x} \ldots c_{k-1} P-\int_{a}^{x} f .
\end{aligned}
$$

\section{REFERENCES}

[1] P.S. Bullen, "A critexion for $n$ convexity", Pacific J. Math. 36 (1971), 81-98.

[2] P.S. Bullen, "The $P^{n}$ integral", J. Austral. Math. Soc. 14(2) (1972), 219-236.

[3] P.S. Bullen and S.N. Mukhopadhyay, "Peano derivatives and general integrals", Pacific J. Math. 47 (1) (1973), 43-58.

[4] P.S. Bullen and S.N. Mukhopodhyay, "Relation between some general $n$th order derivatives", Fund. Math. 85 (1974), 257-276.

[5] E. Corominas, "Contribution a la théorie de la dérivation d'ordre supériour", BulZ. Soc. Math. France 81 (1953), 177-222.

[6] U. Das and A. G. Das, "Convergence in $k$ th variation and $R S_{k}$ integrals", J. Austral. Math. Soc. A 31 (1981), 163-174.

[7] U. Das and A. G. Das, "A new characterisation of $k$-fold Lebesgue. integral", Comment. Math. Prace Mat. 28 (1) (1988), to appear.

[8] A.G. Das and B.K. Lahiri, "On absolutely kth continuous functions", Fund. Math. 105 (1980), 159-169. 
[9] A. Denjoy, "Sur l'integration des co-efficients differentials d'ordre supériour", Fund. Math. 25 (1935), 273-326.

[10] S. De Sarkar and A.G. Das, "On functions of bounded $k$ th variation", J. Indian Inst. Sci. 64 (B), November (1983), 299-309.

[11] S. De Sarkar and A.G. Das, "On functions of bounded essential $k$ th variation", Bull. Calcutta Math. Soc., 78 (1986), 249-258.

[12] R.D. James, "Generalised kth primitives", Trons. Amer. Math. Soc. 76 (1954), 149-176.

[13] H.W. Oliver, "The exact Peano derivative", Trons. Amer. Math. Soc. 76 (1954), 444-456.

[14] J. Ridder, "Uber der Perronscheno Integralbegriff und sine Bezichung zu der $R, L$ und D-Integralen", Math.Z. 34 (1931), 234-269.

[15] A.M. Russell, "Functions of bounded kth variation", Proc. London Math. Soc. $26(3)$ (1973), 547-563.

[16] A.M. Russell, "An integral representation for a generalised variation of a function", Bull. Austral. Math. Soc. 11 (1974), 225-229.

[17] A.M. Russell, "Stieltjes type integrals", J. Austral. Math. Soc.A 20 (1975), 431-448.

[18] A.M. Russell, "Further results on an integral representation of functions of generalised variation", Bull. Austral. Math. Soc. 18 (1978), 407-430.

[19] S. Saks, Theory of the Integral, Warsaw, 1937.

[20] w.L.C. Sargent, "On generalised derivative and Cesaro Denjoy integrals", Proc. London Math. Soc. 52 (2) (1951), 365-376.

[21] S. Verblundky, "On the Peano derivatives", Proc. London Math. Soc. 22 (1971), 313-324.

\section{A.G. Das}

Department of Mathematics

University of Kalyani

Kalyani, Nadia

West Bengal, India.

\section{S. De Sarkar}

Department of Mathematics Nabadwip Vidyasagar College Nadia, West Bengal, India. 